\title{
Vibration Responses and Suppression of Variable Stiffness Laminates with Optimally Steered Fibers and Magnetostrictive Layers
}

\author{
A. H. Akbarzadeh ${ }^{1,2 * \dagger}$, M. Arian $\mathrm{Nik}^{2 *}$, D. Pasini ${ }^{2 \dagger}$ \\ ${ }^{1}$ Department of Bioresource Engineering, McGill University, Ste-Anne-de-Bellevue, Island of Montreal, \\ QC H9X 3V9, Canada \\ ${ }^{2}$ Department of Mechanical Engineering, McGill University, Montreal, QC H3A OC3, Canada
}

\begin{abstract}
This paper examines the vibration of fiber-steered laminated plates, such as those used in the skins of a sandwich panel, manufactured by automated fiber placement. We use third-order shear deformation theory, hybrid Fourier-Galerkin method, and numerical integration technique to predict their vibration responses, and to study the role of manufacturing defects, in particular gaps and overlpas, as well as the parameters representing the stiffness of the sandwich core. With the aim of improving both structural and vibration performance, we first adopt a passive approach to search for optimal fiber paths that can concurrently maximize the undamped dynamic out-of-plane and in-plane stiffness of laminates with gaps and overlaps. To further reduce vibration, we then follow an active approach that uses magnetostrictive layers to suppress the structural vibration of laminates with optimal vibration characteristics. The results of the vibration analysis show that for plates with gaps, as opposed to those with overlaps, the dynamic out-of-plane deflection has a higher amplitude and a lower frequency than that of a defect-free plate. In addition, the results show that magnetostrictive layers with a higher gain control can lead to a lower vibration frequency, and better attenuate the vibration response of the panel.
\end{abstract}

Keywords: B. Defects; A. Laminates; A. Smart materials; B. Vibration; Automated fiber placement.

\section{Introduction}

Compared to single phase metallic and nonmetallic materials, composite materials, including fibrous, particulate, and laminated, offer improved stiffness, strength, fatigue life, corrosion resistance, weight reduction, and self-healing functionality [1-3]. For these reasons, fiber reinforced composites are often preferred in a wide range of applications from aerospace and construction to medicine, among several others [4-6]. Traditionally, laminated fiber-reinforced composites are fabricated by stacking laminae of straight fibers that exhibit the highest strength

\footnotetext{
* A.H. Akbarzadeh (hamid.akbarzadeh@mcgill.ca) and M. Arian Nik (mahdi.ariannik@mail.mcgill.ca) have contributed equally to this work.

† Corresponding authors: hamid.akbarzadeh@mcgill.ca, Tel: +1 (514) 398-7680, Fax: +1 (514) 398-7990 and damiano.pasini@mcgill.ca and, Tel: (+1) 514-398-6295, Fax: (+1) 514-398-7365.
} 
in the fiber direction and the lowest in the direction transverse to the fibers [1, 7]. The recent advent of Automated Fiber Placement (AFP) is contributing to yield a paradigm shift in composite design, i.e. variable stiffness laminates can be built by steering fiber-tows along curvilinear paths [8]. Fiber-steered laminates have been shown to outperform conventional straight-fiber laminates, thereby offering enhanced damage resistance [9], enhanced buckling load [10-14], higher fundamental frequency [15], and improved strength with respect to quasiisotropic laminates [16], besides reduced levels of stress concentration [17]. While these structural improvements are particularly encouraging, manufacturing deviations introduced during the AFP process result in laminates with mechanical properties that differ from those of the original design. In particular, a range of defects, such as gaps and overlaps, appear between the tows, which are resin-rich areas (gaps) and thickness build-ups (overlaps). Recent studies have focused on the effect of gaps and overlaps on the structural properties of steered-fibers laminates. A method introduced in reference [11] to capture the impact of gaps and overlaps on the mechanical properties of fiber-steered composites has been used to achieve improved inplane stiffness and buckling load in thin-walled laminates with embedded gaps and overlaps [18, 19]. A finite element analysis has been also recently carried out to simulate the first-ply failure of tow-steered panels with manufacturing defects [20]. The numerical results revealed how matrix cracking is influenced by gaps between adjacent courses.

Laminated composites with straight fibers are commonly used as skins in sandwich structures $[21,22]$. More recently, fiber-steered composites have been used as the skins of sandwich panels for stowed solar array wings [23]. It has been shown that the localized bending, buckling, and wrinkling of the skins could be approximately modelled by assuming the skin as a plate or shell resting on an elastic foundation that replicates the elastic interaction of the sandwich core [24, 25]. Well-known approaches to model elastic foundations include the Winkler and Pasternak models [26, 27], which are relevant to model the response of a flexible and compressible core of a sandwich panel. Their use in the analysis of sandwich panel responses is often beneficial, as they enable to bypass detailed simulations, where each constituent is individually modelled. In addition, approaches incorporating an elastic foundation could also model the soil-structure interaction of laminated composite slabs in structural systems [28], portable composite bridges on marshy lands [29], and stiffeners of pressurized composite cabins [29]. In this paper, we adopt an elastic foundation model to describe the interaction between the core and skins of a sandwich panel, with the latter made of fiber-steered laminates.

Sandwich panels can also be provided with sensing and actuation functionalities by embedding smart materials into their skins [30, 31]. Improved structural performance, active control of structural vibration, and structural health monitoring are some of the main functionalities that can be obtained with the use of piezoelectric, magnetostrictive, and other active materials. Their use in sandwich panels subjected to vibratory loads has been demonstrated to be effective in attenuating structural vibrations that can lead to catastrophic failure [32]. In general, smart materials are commonly bonded via epoxy-based adhesives to the skins [33]. For example, Terfenol-D, a commercially available magnetostrictive material, has been used for active 
vibration suppression. One of its characteristics is its high energy density and easiness to be embedded into the host materials [34, 35]. Magnetostrictive patches/layers are also broadly used to control noise and vibration of composites panels with constant stiffness [34, 36-38]. On the other hand, the application of smart materials to fiber-steered composites is an area of research currently unexplored, but with a strong potential to reduce vibration. To improve structural and vibration performance, this paper adopts not only a passive approach via optimal fiber steering, but also its active counterpart which involves the integration of magnetostrictive materials.

The paper is organized as follows. Section 2 introduces the geometric parameters used in the equations describing steered fiber trajectories in a laminated composite. Sections 3 and 4 use a third-order shear deformation theory (TSDT) to develop the governing equations for variable stiffness composites with surface-bonded magnetostrictive layers. The TSDT theory is chosen to provide a general framework that models skins with both thin and moderately-thick thickness [39-49]. The advantage of TSDT is the relaxation of the kinematic assumptions on the straightness and normality of the transverse plane, thereby avoiding the use of any shear correction factor. Sections 5.1 to 5.3 describe a passive approach to vibration reduction, with particular focus on the search of optimal fiber paths in the skins that simultaneously minimize vibration and maximize the panel stiffness. Finally, the active approach is presented in Section 5.4, where magnetostrictive layers are bonded to the skins with the optimal steered-fibers found in section 5, to gage whether smart materials can further improve panel vibrations.

\section{Fiber-steered laminated composite}

An AFP machine is capable of combining tape placement and filament winding by placing on a mould a band of tows, called a "course", of prepreg composites along a predefined path [11, 20, 50]. To model a fiber-steered laminate, we define here a reference path along which the AFP machine places the first course. Subsequent fiber paths are obtained by shifting the reference path along a given direction, e.g. $x$ - or $y$-directions [51]. A reference fiber path can be defined by a continuous and smooth mathematical function. A complex function can offer high flexibility for laminate tailoring; this, however, increases the number of design variables and comes with an additional computational cost. Moreover, highly curved paths modelled with a complex function

might not be necessarily manufacturable with AFP due to the likelihood of violating the constraint imposed to the minimum turning radius. In this study, we opt for a constant curvature fiber path [52] with a trajectory given by

$$
\begin{gathered}
\cos \theta=\cos T_{0}+\frac{|x|}{R} \\
R=\left|\frac{a / 2}{\cos \left(T_{1}\right)-\cos \left(T_{0}\right)}\right|
\end{gathered}
$$


where $\theta$ represents the fiber orientation along the fiber path, $T_{0}$ is the fiber orientation at the plate center, $T_{1}$ is the fiber orientation at the plate edges, $R$ is the turning radius, and |.| denotes the absolute value. The curvature $k$ of the fiber path is also defined by $k=1 / R$. A single layer with this fiber path definition may be represented by $\left\langle T_{0} \mid T_{1}\right\rangle$, where $T_{0}=T_{1}$ represents a straight fiber. The shifting direction of the reference fiber path, affecting both fiber angle and defect distribution, plays a major role on the maximum performance that can be achieved by fibersteering [53]. Figure 1 depicts the impact of the shifting direction on both the fiber angle and gap distribution within a $<45 \mid 26>$ layup, chosen here as an example. For more details on the effect of the course offset direction, interested readers may refer to [53, 54].
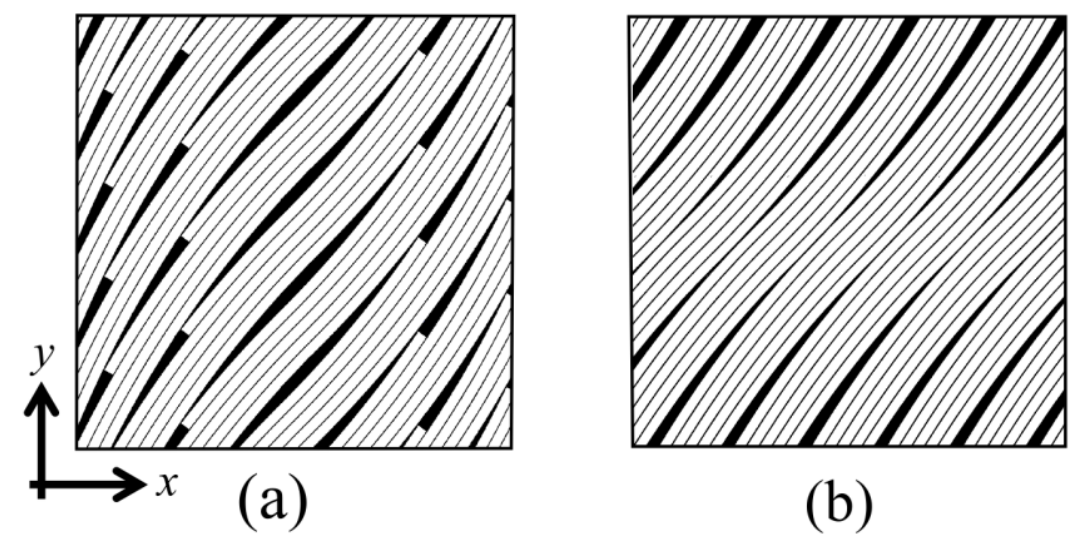

(b)

Fig. 1. Gap (shaded area) distribution; (a) fibers are shifted along the $y$-direction and (b) fibers are shifted along the $x$-direction.

\section{Problem definition and governing equations}

We consider a rectangular sandwich plate with fiber-steered skins and assess the role of a range of parameters describing fiber-steering, manufacturing defects, and elastic stiffness of the sandwich core. The compressibility and shear deformation of the sandwich core, which can be made for example of polyurethane foam, are described by the spring stiffness $k_{w}$ and the shear layer stiffness $k_{s}$, which are commonly used in the analysis of an elastic foundation with a Pasternak model [55]. A dynamic distributed transverse load $q(x, y, t)$ is applied, as a step function, to the composite plate. Furthermore, we introduce magnetostrictive patches fully bonded to the surface of the fiber-steered laminates away from the neutral axis [1,34], and assess their impact on the following responses: linear forced-vibration, time harmonic, and vibration suppression control. Figure $2 \mathrm{a}$ shows four patches distributed in the $x$ and $y$ directions with respective spacing $x_{M}$ and $y_{M}$. While the number, shape, and size of the patches can vary, here we simplify the current analysis by considering the skin of a sandwich with attached magnetostrictive layers covering the entire surfaces of the panel, as shown in Fig 2b. The 
thickness of the composite laminate and the magnetostrictive layers are $h$ and $h_{M}$, respectively, and the plate dimensions, i.e. length, width, and the total thickness, are $a, b$, and $h+2 h_{M}$ (Fig. $2 b)$.

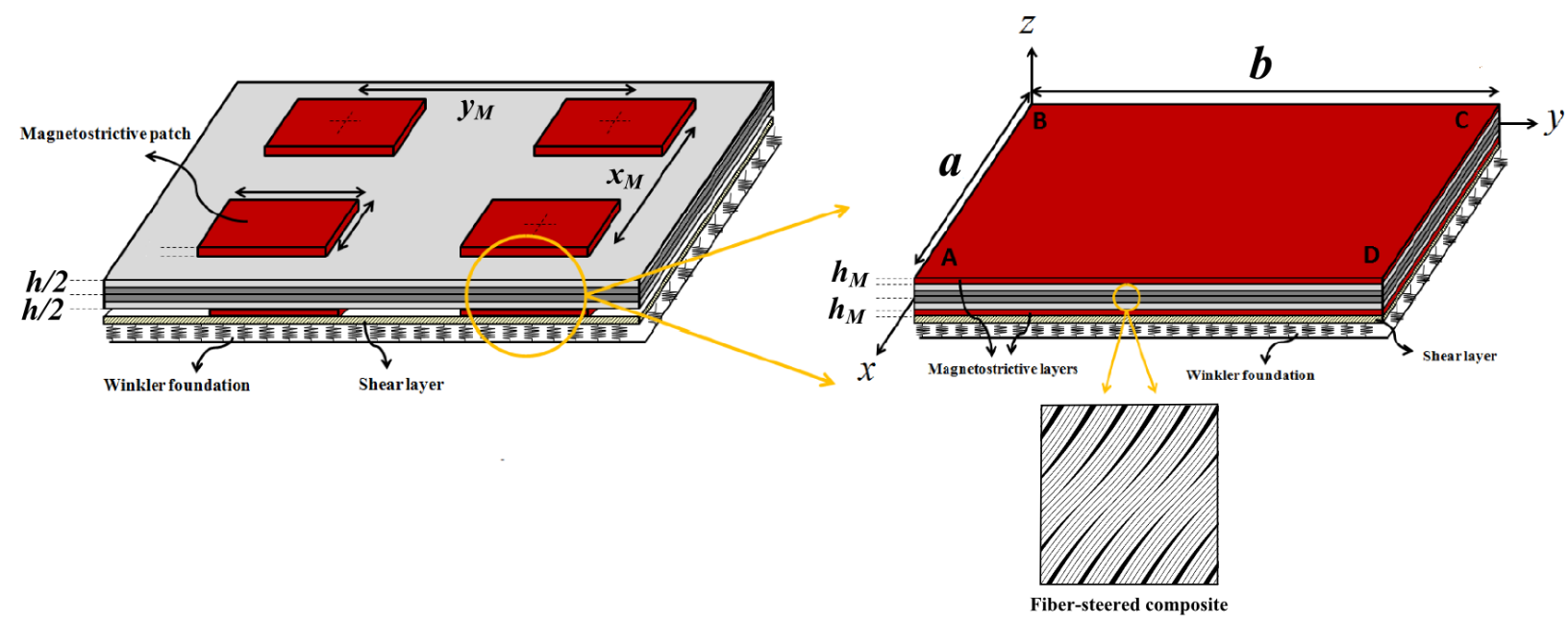

(a)

(b)

Fig. 2. Rectangular variable stiffness plate resting on an elastic foundation with: (a) magnetostrictive patches; (b) magnetostrictive layers covering the whole panel and bonded to both the outer skin surfaces. Simply-supported boundary conditions are specified along the edges ABCD.

To accurately predict the kinematic behavior of moderately-thick plates with variable stiffness, the displacement field $(u, v, w)$, along the $(x, y, z)$ coordinate axes, is expressed via TSDT as [1]:

$$
\begin{gathered}
u(x, y, z, t)=u_{0}(x, y, t)+z \phi_{x}(x, y, t)-c_{1} z^{3}\left(\phi_{x}(x, y, t)+\frac{\partial w_{0}(x, y, t)}{\partial x}\right) \\
v(x, y, z, t)=v_{0}(x, y, t)+z \phi_{y}(x, y, t)-c_{1} z^{3}\left(\phi_{y}(x, y, t)+\frac{\partial w_{0}(x, y, t)}{\partial y}\right) \\
w(x, y, z, t)=w_{0}(x, y, t)
\end{gathered}
$$

where $\left(u_{0}, v_{0}, w_{0}\right)$ represents the displacement components of the midplane $(z=0)$. Moreover, $\phi_{x}$ and $\phi_{y}$ stand for the rotations about the $y$ - and $x$-axes, $t$ represents time, and $c_{1}=\frac{4}{3\left(h+2 h_{M}\right)^{2}}$. Substituting the displacement field, Eq. (2), into the linear strain-displacement relations results in $[1,34]$ : 


$$
\begin{gathered}
\left\{\begin{array}{l}
\varepsilon_{x x} \\
\varepsilon_{y y} \\
\gamma_{x y}
\end{array}\right\}=\left\{\begin{array}{c}
u_{0, x} \\
v_{0, y} \\
u_{0, y}+v_{0, x}
\end{array}\right\}+z\left\{\begin{array}{c}
\varphi_{x, x} \\
\varphi_{y, y} \\
\varphi_{x, y}+\varphi_{y, x}
\end{array}\right\}-c_{1} z^{3}\left\{\begin{array}{c}
\varphi_{x, x}+w_{0, x x} \\
\varphi_{y, y}+w_{0, y y} \\
\varphi_{x, y}+\varphi_{y, x}+2 w_{0, x y}
\end{array}\right\} \\
\left\{\begin{array}{l}
\gamma_{y z} \\
\gamma_{x z}
\end{array}\right\}=\left(1-c_{2} z^{2}\right)\left\{\begin{array}{l}
\varphi_{y}+w_{0, y} \\
\varphi_{x}+w_{0, x}
\end{array}\right\}
\end{gathered}
$$

where $c_{2}=3 c_{1}$, and the normal and shear strains are represented by $\left(\varepsilon_{x x}, \varepsilon_{y y}\right)$ and $\left(\gamma_{x y}, \gamma_{x z}, \gamma_{y z}\right)=2\left(\varepsilon_{x y}, \varepsilon_{x z}, \varepsilon_{y z}\right)$; comma stands for the partial differentiation operator.

The TSDT equations of motion for a plate resting on a Pasternak elastic foundation are derived by the principle of the virtual work $[1,26,56]$ :

$$
\begin{gathered}
N_{x x, x}+N_{x y, y}=I_{0} \ddot{u}_{o}+J_{1} \ddot{\varphi}_{x}-c_{1} I_{3} \ddot{w}_{0, x} \\
N_{x y, x}+N_{y y, y}=I_{0} \ddot{v}_{o}+J_{1} \ddot{\varphi}_{y}-c_{1} I_{3} \ddot{w}_{0, y} \\
\bar{Q}_{x, x}+\bar{Q}_{y, y}+c_{1}\left(P_{x x, x x}+2 P_{x y, x y}+P_{y y, y y}\right)+q(x, y, t)-k_{w} w_{0}+k_{s}\left(w_{0, x x}+w_{0, y y}\right)=I_{0} \ddot{w}_{o}-c_{1}^{2} I_{6}\left(\ddot{w}_{0, x x}+\ddot{w}_{0, y y}\right) \\
+c_{1}\left(I_{3}\left(\ddot{u}_{0, x}+\ddot{v}_{0, y}\right)+J_{4}\left(\ddot{\varphi}_{x, x}+\ddot{\varphi}_{y, y}\right)\right) \\
\bar{M}_{x x, x}+\bar{M}_{x y, y}-\bar{Q}_{x}=J_{1} \ddot{u}_{o}+K_{2} \ddot{\varphi}_{x}-c_{1} J_{4} \ddot{w}_{0, x} \\
\bar{M}_{x y, x}+\bar{M}_{y y, y}-\bar{Q}_{y}=J_{1} \ddot{v}_{o}+K_{2} \ddot{\varphi}_{y}-c_{1} J_{4} \ddot{w}_{0, y}
\end{gathered}
$$

where

$$
\begin{array}{r}
\left(N_{\alpha \beta}, M_{\alpha \beta}, P_{\alpha \beta}\right)=\int_{-\frac{h}{2}-h_{M}}^{\frac{h}{2}+h_{M}} \sigma_{\alpha \beta}\left(1, z, z^{3}\right) d z,\left(Q_{\alpha}, R_{\alpha}\right)=\int_{-\frac{h}{2}-h_{M}}^{\frac{h}{2}+h_{M}} \sigma_{\alpha z}\left(1, z^{2}\right) d z, \bar{M}_{\alpha \beta}=M_{\alpha \beta}-c_{1} P_{\alpha \beta} \\
\bar{Q}_{\alpha}=Q_{\alpha}-c_{2} R_{\alpha}, I_{i}=\int_{-\frac{h}{2}-h_{M}}^{\frac{h}{2}+h_{M}} \rho z^{i} d z, J_{i}=I_{i}-c_{1} I_{i+2}, K_{2}=I_{2}-2 c_{1} I_{4}+c_{1}^{2} I_{6} \\
(\alpha, \beta=x, y)(i=0,1, \ldots, 6)
\end{array}
$$


and $\sigma_{\alpha \beta}$ and $\rho$ are, respectively, the stress components and mass density; the superposed dot on a variable stands for the time derivative. We note that TSDT governing equations include FSDT and CLPT formulations as special cases $[1,48]$.

The stress resultants $N, M, P, Q$, and $R$ of the actively controlled composite plate are related to the strain and displacement fields as $[1,34]$ :

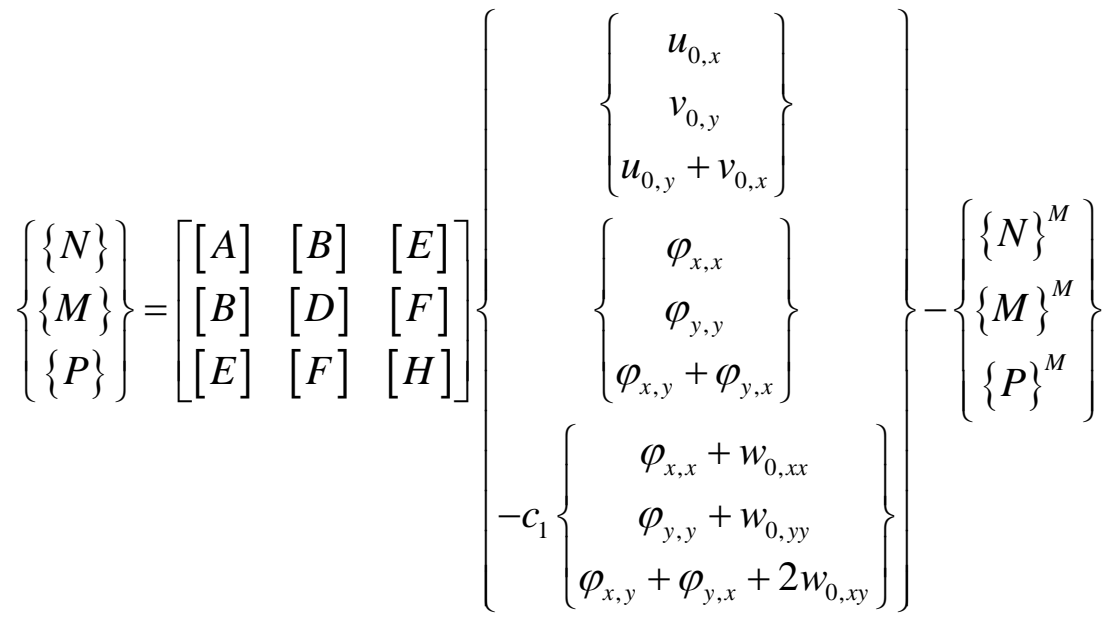

$$
\begin{aligned}
& \left\{\begin{array}{l}
\{Q\} \\
\{R\}
\end{array}\right\}=\left[\begin{array}{ll}
{[A]} & {[D]} \\
{[D]} & {[F]}
\end{array}\right]\left\{\begin{array}{c}
\left\{\begin{array}{l}
\varphi_{y}+w_{0, y} \\
\varphi_{x}+w_{0, x}
\end{array}\right\} \\
-c_{2}\left\{\begin{array}{l}
\varphi_{y}+w_{0, y} \\
\varphi_{x}+w_{0, x}
\end{array}\right\}
\end{array}\right\}
\end{aligned}
$$

The components of $3 \times 3$ stiffness matrices are [57-59]:

$$
\left(A_{k l}, B_{k l}, D_{k l}, E_{k l}, F_{k l}, H_{k l}\right)=\int_{-\frac{h}{2}-h_{M}}^{\frac{h}{2}+h_{M}} \bar{Q}_{k l}\left(1, z, z^{2}, z^{3}, z^{4}, z^{6}\right) d z \quad(k, l=1,2,6)
$$

where $\overline{Q_{i j}}$ is the components of the transformed plane stress-reduced stiffness matrix. The actuation stress resultants of the magnetostrictive layers presented in Eq. (6), $\{N\}^{M},\{M\}^{M}$, and $\{P\}^{M}$, are defined as $[1,60,61]$ :

$$
\left\{\begin{array}{l}
N_{x x}^{M} \\
N_{y y}^{M} \\
N_{x y}^{M}
\end{array}\right\}=\int_{-\frac{h}{2}-h_{M}}^{-h_{M}} H_{z}^{\left(M_{\text {Botom }}\right)}\left\{\begin{array}{l}
\bar{d}_{31} \\
\bar{d}_{32} \\
\bar{d}_{36}
\end{array}\right\} d z+\int_{\frac{h}{2}}^{\left(M_{\text {Botom }}\right)} H_{z}^{\left(M_{\text {Top }}\right)}\left\{\begin{array}{l}
\bar{d}_{31} \\
\bar{d}_{32} \\
\bar{d}_{36}
\end{array}\right\} d z
$$




$$
\begin{aligned}
& \left\{\begin{array}{l}
M_{x x}^{M} \\
M_{y y}^{M} \\
M_{x y}^{M}
\end{array}\right\}=\int_{-\frac{h}{2}-h_{M}}^{-h_{M}} H_{z}^{\left(M_{\text {Botom }}\right)}\left\{\begin{array}{l}
\bar{d}_{31} \\
\bar{d}_{32} \\
\bar{d}_{36}
\end{array}\right\}^{\left(M_{\text {Botom }}\right)} z d z+\int_{\frac{h}{2}}^{\frac{h}{2}+h_{M}} H_{z}^{\left(M_{\text {Top }}\right)}\left\{\begin{array}{l}
\bar{d}_{31} \\
\bar{d}_{32} \\
\bar{d}_{36}
\end{array}\right\}^{\left(M_{\text {Top }}\right)} z d z \\
& \left\{\begin{array}{l}
P_{x x}^{M} \\
P_{y y}^{M} \\
P_{x y}^{M}
\end{array}\right\}=\int_{-\frac{h}{2}-h_{M}}^{-h_{M}} H_{z}^{\left(M_{\text {Botom }}\right)}\left\{\begin{array}{l}
\bar{d}_{31} \\
\bar{d}_{32}^{\left(M_{\text {Botom }}\right)} \\
\bar{d}_{36}
\end{array}\right\} z^{2} d z+\int_{\frac{h}{2}}^{\frac{h}{2}+h_{M}} H_{z}^{\left(M_{\text {Top }}\right)}\left\{\begin{array}{l}
\bar{d}_{31} \\
\bar{d}_{32} \\
\bar{d}_{36}
\end{array}\right\}^{\left(M_{\text {Top }}\right)} z^{2} d z
\end{aligned}
$$

where $H_{z}$ is the magnetic field intensity; $d_{31}, d_{32}$, and $d_{36}$ are the magnetostrictive/piezomagnetic coefficients of the smart material layers at the top $\left(M_{T o p}\right)$ and bottom ( $\left.M_{\text {Bottom }}\right)$ of the plate (Fig. 2b).

Magnetostrictive materials contribute to the vibration control via a velocity dependent feedback law that governs the current of the magnetic coils, which activate the magnetostrictive materials [62]. For velocity-proportional, and closed-loop feedback control, the magnetic field intensity $\left(H_{z}\right)$ produced by the coil is expressed in terms of the coil current $I(x, t)$ as [34,63]:

$$
H_{z}(x, y, t)=K_{C} I(x, t)
$$

and $I(x, t)$ is obtained as a function of the out-of-plane deflection velocity $\dot{w}_{0}$ through [34]:

$$
I(x, t)=C \dot{w}_{0}
$$

where $K_{C}$ is the coil constant and $C$ is the gain control. The coil constant depends on the coil width and radius, and the number of turns of the coil $[1,64]$.

Substituting Eqs. (6) through (10) into the equation of motion (4) leads to five coupled differential equations that govern the response of a thin and moderately-thick fiber-steered plate. We note that the governing equations are derived here for a specially orthotropic $\left(D_{16}=D_{26}=F_{16}=F_{26}=H_{16}=H_{26}=0\right)$ laminated composite with a balanced $\left(A_{16}=A_{26}=0\right)$ and symmetric $\left(B_{i j}=E_{i j}=0\right)$ layup.

\section{Methodology}

In contrast to constant stiffness composites, the stiffness matrix of a fiber-steered plate depends on the spatial coordinates $(x, y)$. As a result, the development of closed-form solutions of 
vibration response for variable stiffness composites is cumbersome. Here, we use a semi-analytic methodology [48] for a plate with simply-supported boundary conditions (SS-1) [1, 64].

The displacement fields can be expressed in terms of Fourier series expansion to satisfy the boundary conditions [57]:

$$
\left\{\begin{array}{l}
u_{0}(x, y, t) \\
v_{0}(x, y, t) \\
w_{0}(x, y, t) \\
\phi_{x}(x, y, t) \\
\phi_{y}(x, y, t)
\end{array}\right\}=\sum_{n=1}^{n_{y}} \sum_{m=1}^{m_{x}}\left\{\begin{array}{c}
U_{m n}(t) \cos \left(r_{m} x\right) \sin \left(r_{n} y\right) \\
V_{m n}(t) \sin \left(r_{m} x\right) \cos \left(r_{n} y\right) \\
W_{m n}(t) \sin \left(r_{m} x\right) \sin \left(r_{n} y\right) \\
X_{m n}(t) \cos \left(r_{m} x\right) \sin \left(r_{n} y\right) \\
Y_{m n}(t) \sin \left(r_{m} x\right) \cos \left(r_{n} y\right)
\end{array}\right\}
$$

where $r_{m}=\frac{m \pi}{a}, r_{n}=\frac{n \pi}{b}$, and $n_{y}$ and $m_{x}$ are arbitrary integers for summation. The unknown coefficients $U_{m n}, V_{m n}, W_{m n}, X_{m n}$, and $Y_{m n}$ could be determined by using the displacement field Eq. (11) and enforcing the residual to be orthogonal to given weight functions [1]. The governing differential equations could be solved via the semi-analytic Fourier-Galerkin method [48]:

$$
\int_{y=0}^{b} \int_{x=0}^{a}\left\{\begin{array}{l}
R_{1}\left(U_{m n}, V_{m n}, W_{m n}, X_{m n}, Y_{m n}\right) \cos \left(r_{p} x\right) \sin \left(r_{q} y\right) \\
R_{2}\left(U_{m n}, V_{m n}, W_{m n}, X_{m n}, Y_{m n}\right) \sin \left(r_{p} x\right) \cos \left(r_{q} y\right. \\
R_{3}\left(U_{m n}, V_{m n}, W_{m n}, X_{m n}, Y_{m n}\right) \sin \left(r_{p} x\right) \cos \left(r_{q} y\right. \\
R_{4}\left(U_{m n}, V_{m n}, W_{m n}, X_{m n}, Y_{m n}\right) \sin \left(r_{p} x\right) \cos \left(r_{q} y\right. \\
R_{5}\left(U_{m n}, V_{m n}, W_{m n}, X_{m n}, Y_{m n}\right) \sin \left(r_{p} x\right) \cos \left(r_{q} y\right.
\end{array}\right\} d x d y=0 \quad\left(p=1,2, \ldots, m_{x}, q=1,2, \ldots, n_{y}\right)
$$

where $R_{i}(i=1, \ldots, 5)$ represent the residual of each governing differential equation. The resulting $\left(5 \times m_{x} \times n_{y}\right) \times\left(5 \times m_{x} \times n_{y}\right)$ system of differential equations could be written as:

$$
\left[K_{T S D T}\right]\left\{\Delta_{T S D T}\right\}+\left[C_{T S D T}\right]\left\{\dot{\Delta}_{T S D T}\right\}+\left[M_{T S D T}\right]\left\{\ddot{\Delta}_{T S D T}\right\}=\left\{F_{T S D T}\right\}
$$

where $K_{T S D T}, C_{T S D T}$, and $M_{T S D T}$ are the stiffness, damping, and mass matrices, respectively; $F_{T S D T}$ represents the mechanical force vector and $\left\{\Delta_{T S D T}\right\}^{T}=\left\{\begin{array}{llllllllllll}U_{11} & V_{11} & W_{11} & X_{11} & Y_{11} & \ldots & U_{m_{x} n_{y}} & V_{m_{x} n_{y}} & W_{m_{x} n_{y}} & X_{m_{x} n_{y}} & Y_{m_{x} n_{y}}\end{array}\right\} . \quad$ The damping matrix, $C_{T S D T}$, vanishes in the absence of the active magnetostrictive layers and internal structural damping.

For forced-vibration analysis, the Newmark integration procedure is used to solve Eq. (13) in the time domain [1]. For vibration control, the force vector $F_{T S D T}$ is neglected in Eq. (13) and the solutions for Eq. (13) are sought in the form of [34, 65]: 


$$
\left\{\Delta_{T S D T}\right\}=\left\{\bar{\Delta}_{T S D T}\right\} e^{\lambda t}
$$

where $\lambda$ is obtained for non-trivial solutions by setting the following determinant to zero:

$$
\left|\left[K_{T S D T}\right]+\left[C_{T S D T}\right] \lambda+\left[M_{T S D T}\right] \lambda^{2}\right|=0
$$

The lowest eigenvalue of Eq. (15), in the form of $\lambda=-\alpha+i \omega_{d}$, corresponds to the out-of-plane deflection. It is worthwhile to note that $\alpha=\zeta \omega_{n}$ and $\zeta, \omega_{n}$, and $\omega_{d}$ are, respectively, the damping ratio, the undamped angular natural frequency, and the damped angular frequency as the characteristics of the suppressed vibration. Furthermore, the harmonic responses could be obtained by substituting [66, 67]

$$
\left\{\Delta_{T S D T}\right\}=\left\{\bar{\Delta}_{T S D T}\right\} e^{\omega t},\left\{F_{T S D T}\right\}=\left\{\bar{F}_{T S D T}\right\} e^{\omega t}
$$

into Eq. (13) and solving the resulting algebraic equation for a range of harmonic angular frequencies, $\omega$. MATLAB scripts have been developed to implement the abovementioned procedure for the vibration analysis of a fiber-steered plate with embedded defects.

\section{Results and discussion}

The methodology described above is applied to a simply-supported, moderately-thick, fibersteered composite plate, such as that used for the skin of a sandwich panel (Fig. 2). The plate has square geometry $(a=b=1 \mathrm{~m})$ with a symmetric composite layup, and it is subjected to a uniformly distributed transverse load, represented by $q_{0}(x, y, t)=q_{0}(t)$. The results described here focus on a number of factors; in particular the role of manufacturing defects, i.e. gaps and overlaps, fiber orientation, stiffness parameters of the sandwich core, and length-to-thickness ratio of the plate on the undamped forced-vibration (Sections 5.1) and time-harmonic responses (Section 5.2). In addition, we present results for the optimum fiber paths of fiber steered skins that maximize the dynamic out-of-plane and static in-plane properties of a sandwich panel under multiple load cases (Section 5.3). We finally turn our attention to the integration of the magnetostrictive layers on the characteristics of the damped responses of an optimal fiber-steered layup (Section 5.4).

In presenting the results for the out-of-plane deflection, out-of-plane velocity, and response frequency, we make use of the following non-dimensional terms: $\bar{w}=w_{0} \frac{E_{2}^{c} h^{3}}{q_{0} a^{4}}, \overline{\dot{w}}=\dot{w}_{0} \frac{\sqrt{\rho^{c} E_{2}^{c}}}{q_{0}}$, and $\bar{\omega}=\omega \frac{a^{2}}{h} \sqrt{\frac{\rho^{c}}{E_{2}^{c}}}$, where the superscript "c" stands for composite and the subscript "2" 
represents the material properties along $y$-axis, with values for fiber and resin given in reference [48].

\subsection{Forced-vibration response}

For the undamped/uncontrolled $\left(h_{M}=0\right)$ forced-vibration responses, we examine the effect of manufacturing parameters, elastic foundation constants, and fiber orientation for a plate with length-to-thickness ratio of $a / h=10$. A 16-ply balanced and symmetric fiber-steered laminate with a $[ \pm<58 \mid 39>]_{4 s}$ layup is considered to validate the numerical results with those given in reference [48].

Role of manufacturing defects. Figure 3 a merely shows the undamped temporal deflection at the plate midpoint, where the maximum out-of-plane deflection of the fiber-steered plate occurs. It can be seen that the panel made with a complete-gap strategy has a higher amplitude of dynamic deflection and a lower response frequency than that of a defect-free panel. A complete overlap strategy, on the other hand, leads to a lower amplitude of deflection and a higher frequency. For example, the deflection amplitude increases of $9 \%$ and the frequency decreases of $3 \%$ for a plate with gaps compared to a defect-free plate, whereas the deflection amplitude for a plate with overlaps decreases of $24 \%$ and the frequency increases of $10 \%$. These observations are in agreement with the results given in reference [48] for static deflection and natural frequency. We attribute to the morphology of manufacturing defects the cause for the changes in the dynamic responses. Gaps are resin-rich areas with lower stiffness, while overlaps are thickness build-ups with improved flexural stiffness.

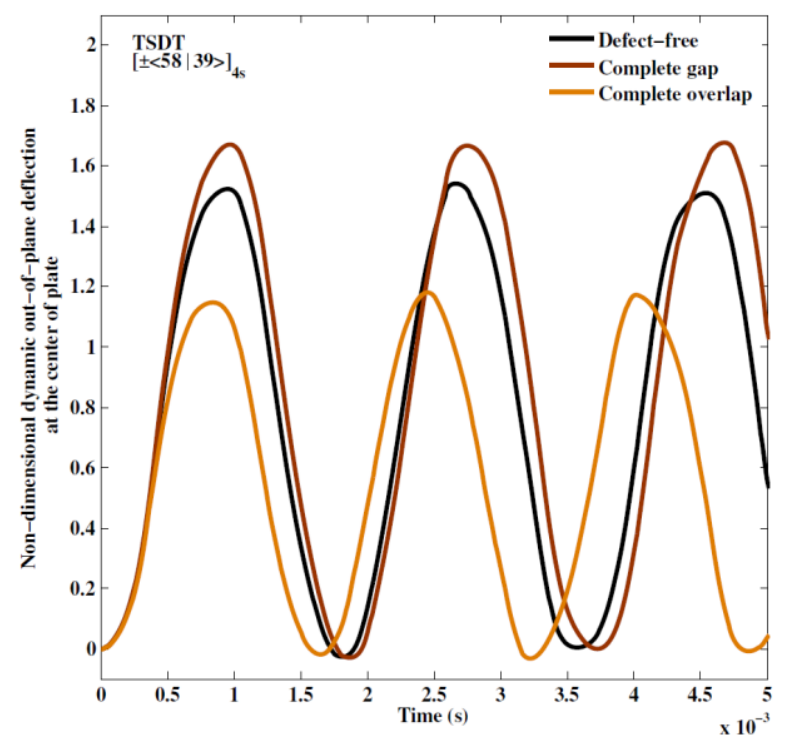

(a)

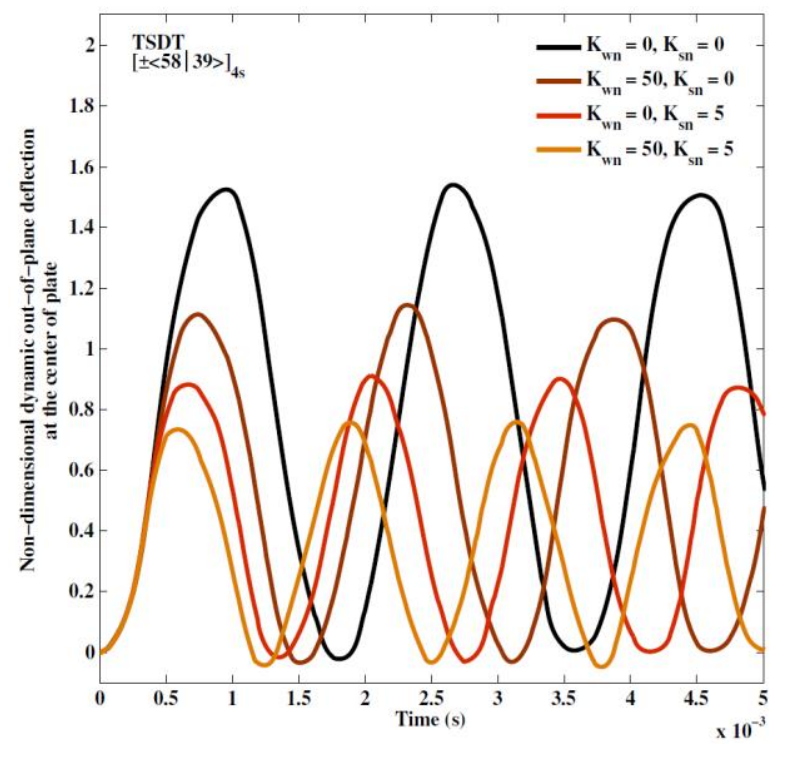

(b) 
Fig. 3 Dynamic out-of-plane deflection $(\bar{w} \times 100)$ at the center of a variable stiffness plate for (a) alternative manufacturing strategies and (b) alternative elastic foundation for a defect-free design.

Role of core stiffness constants. Figure $3 \mathrm{~b}$ shows the role of the elastic foundation parameters on the undamped temporal deflection for a defect-free variable stiffness plate. The time evolution of the midpoint out-of-plane deflection is plotted for alternative elastic stiffness, given in nondimensional forms as: $K_{w n}=\frac{k_{w} a^{4}}{D_{c}}$ and $K_{s n}=\frac{k_{s} a^{2}}{D_{c}}$, where $D_{c}=\frac{E_{c} h^{3}}{12\left(1-v_{c}{ }^{2}\right)}$ is the flexural rigidity of a constant stiffness composite plate. The dynamic responses are compared in Fig. $3 \mathrm{~b}$ for four alternative elastic foundations: $\left(K_{w n}, K_{s n}\right)=(0,0)$ (Unconstrained), $\left(K_{w n}, K_{s n}\right)=(50,0)$ (Winkler foundation), $\left(K_{w n}, K_{s n}\right)=(0,5) \quad$ (Shear layer foundation), and $\left(K_{w n}, K_{s n}\right)=(50,5)$ (Pasternak foundation) [68]. The presence of an elastic foundation, caused by the compressible support of the core material in a sandwich panel, decreases the amplitude of the dynamic out-ofplane deflection and increases the frequency responses due to the increased out-of-plane stiffness of the structure. We observe that the shear layer parameter $\left(k_{s}\right)$ has a more pronounced effect on the dynamic responses than the Winkler parameter $\left(k_{w}\right)$. A Winkler elastic foundation $\left(K_{w n}, K_{s n}\right)=(50,0)$ decreases the amplitude of the dynamic deflection by $27 \%$ and enhances the response frequency by $19 \%$ compared to the unconstrained plate. For a shear layer elastic foundation $\left(K_{w n}, K_{s n}\right)=(0,5)$, the dynamic deflection decreases of $42 \%$ and the frequency increases of $35 \%$.

To show the potential of a variable stiffness design in improving the dynamic out-of-plane response of a composite panel, we plot in Fig. 4 the response domains for deflection amplitude and frequency versus the curvature of a curvilinear fiber path $(k)$. Considering $0.635 \mathrm{~m}(25 \mathrm{in})$ as the minimum turning radius of the fiber path imposed as a manufacturing constraint by a typical AFP machine, the numerical results are limited to curvatures $0 \leq k \leq 1.57\left(\mathrm{~m}^{-1}\right)$. We recall that $k=0$ corresponds to a straight fiber path, which represents a constant stiffness plate. In Fig. 4 the boundaries between the upper and lower bounds for both dynamic deflection $(w)$ and frequency $(\omega)$ appear for $k=0$ and $k=1.57$ respectively. The domains show that a complete gap manufacturing strategy shifts the response domain towards a dynamic deflection with higher amplitude and a lower response frequency compared to the defect-free strategy, due to the lower stiffness of gaps, which are resin-rich areas. In contrast, overlaps shift the response domain towards a lower deflection and a higher frequency, as overlaps are thickness build-ups that stiffen the composite plate along the fiber path. In addition, the boundaries of the domain responses are dependent on the manufacturing strategy, besides the curvature of fiber path. For defect-free fiber-steered composites with $k=1.57$, the boundaries of the dynamic deflection and frequency response are, respectively, $69.3 \%$ and $35.7 \%$ lower than those for $k=0$. 


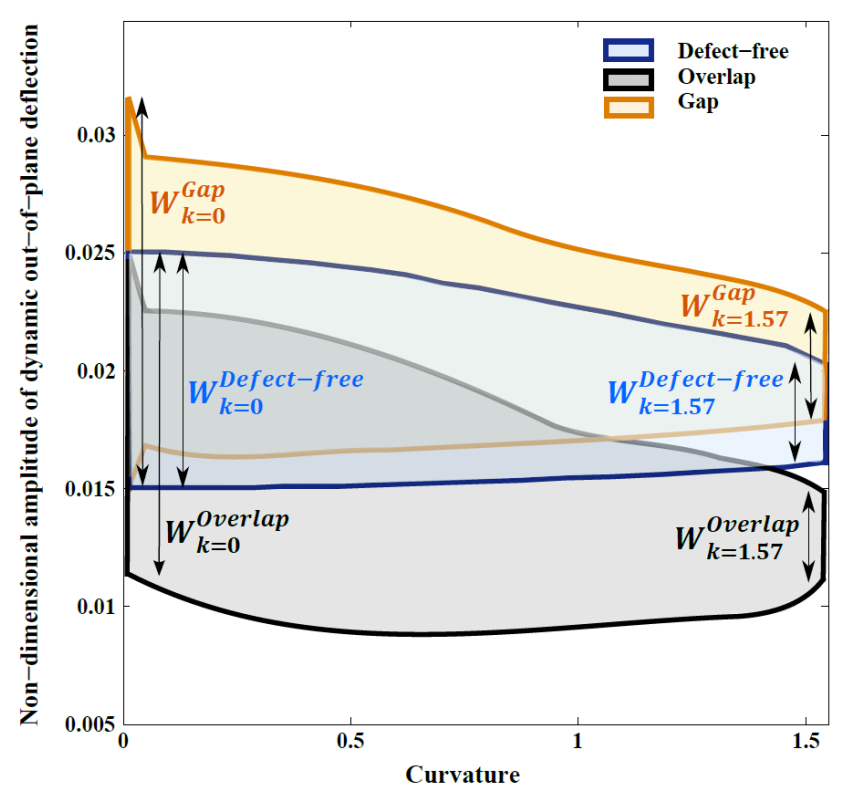

(a)

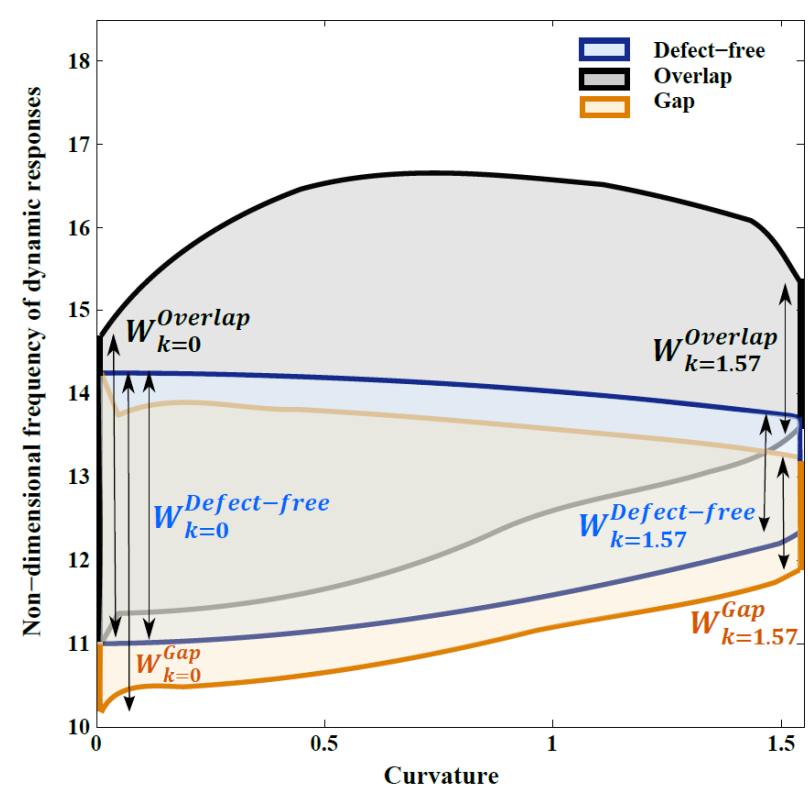

(b)

Fig. 4 Dynamic response domains of a variable stiffness laminate: (a) amplitude of out-of-plane deflection $(\bar{w} \times 100)$ at the center of plate and (b) dynamic response frequency. The boundaries for deflection and frequency are:

$$
\begin{gathered}
w_{k=0}^{\text {Defect-free }}=0.0101, w_{k=0}^{\text {Overlap }}=0.0137, w_{k=0}^{\text {Gap }}=0.0166, w_{k=1.57}^{\text {Defect-free }}=0.0036, w_{k=1.57}^{\text {Overlap }}=0.0042, w_{k=1.57}^{\text {Gap }}=0.0047, \\
\omega_{k=0}^{\text {Defect-free }}=3.26, \omega_{k=0}^{\text {Overlap }}=2.72, \omega_{k=0}^{\text {Gap }}=4.08, \omega_{k=1.57}^{\text {Defect-free }}=1.38, \omega_{k=1.57}^{\text {Overlap }}=1.75, \text { and } \omega_{k=1.57}^{\text {Gap }}=1.36 .
\end{gathered}
$$

\subsection{Harmonic responses}

This section examines the undamped $\left(h_{M}=0\right)$ harmonic responses of a fiber-steered composite plate with a layup $[ \pm<58 \mid 39>]_{4 s}$. The amplitude of the midpoint deflection (w), normalized by the static deflection ( $\mathbf{w}_{\mathbf{0}}$ ), is plotted in $\mathrm{dB}$ for a high frequency range of a harmonic, out-of-plane load.

Figure 5 shows the first-mode $(m=n=1)$ harmonic responses of a variable stiffness plate with $a / h=10$ for three manufacturing scenarios, i.e. defect-free, complete gap, and complete overlap. For each of them, three peaks corresponding to the resonance frequencies are detected, i.e. out-of-plane deflection $\left(w_{0}\right)$ and rotation about the $y-\left(\varphi_{x}\right)$ and $x$ - $\left(\varphi_{y}\right)$ axes. We recall that the in-plane displacements $\left(u_{0}, v_{0}\right)$ are zero for a symmetric layup and simply-supported boundary conditions. As mentioned in Section 5.1, a plate with gaps has a lower stiffness and a plate with overlaps has a higher stiffness compared to a defect-free plate. As a result, the first resonance frequency $\left(w_{0}\right)$ of a plate with gaps is lower than that of a defect-free plate, as opposed to a plate with overlaps which has a higher resonance frequency. In contrast, the 
aforementioned trends are reverse for the second $\left(\varphi_{y}\right)$ and third $\left(\varphi_{x}\right)$ resonance frequency. We recall that CLPT neglects the rotational resonance frequencies, whereas TSDT can predict transverse, translational, and rotational resonance frequencies. Since the rotational resonance occurs at very high frequencies, the use of TSDT becomes relevant in applications involving structural health monitoring of fiber-steered composites [69].

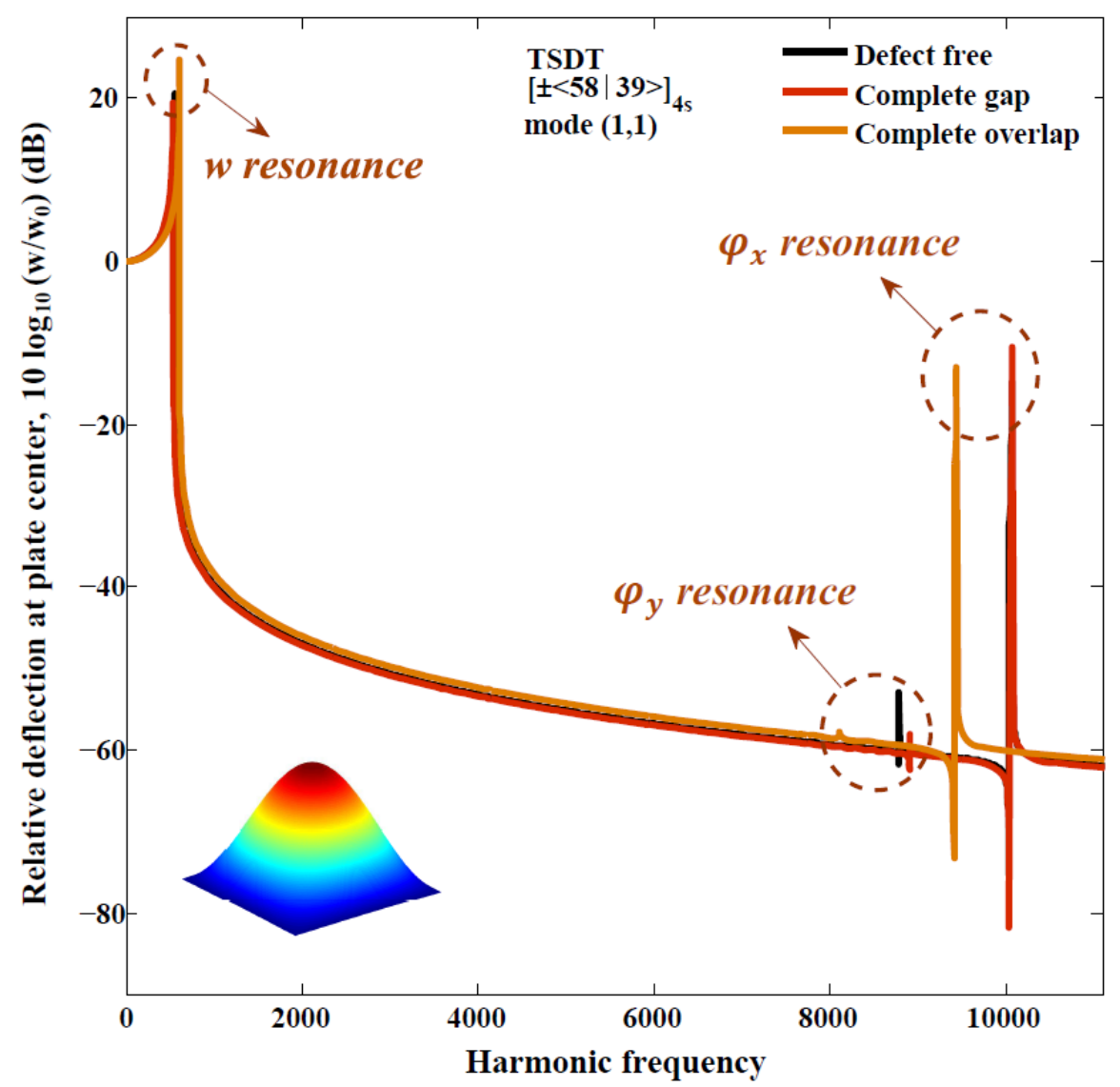

Fig. 5 Effect of manufacturing strategies on harmonic responses.

To isolate the effect of the harmonic mode, length-to-thickness ratio, and elastic foundation constants from the manufacturing defects, Fig. 6 depicts the harmonic responses of a defect-free variable stiffness plate. As shown in Fig. 6a, an increase of the mode number of the harmonic responses enhances all the resonance frequencies, including transverse and rotational, as well as the relative deflection. Furthermore, an increase of the length-to-thickness ratio of the plate reduces the transverse resonance frequency, a result in agreement with the behaviour observed in reference [48], and increases the rotational resonance frequencies. For instance, increasing the length-to-thickness ratio from $a / h=5$ to $a / h=10$ decreases the transverse resonance frequency by $34 \%$ and increases the rotational frequency $\varphi_{y}$ by $92 \%$. Figure $6 \mathrm{c}$ also reveals the dominant effect of the elastic foundation on the transverse resonance frequency, compared to the 
rotational one. While increasing the foundation parameters $K_{w n}$ and $K_{s n}$ significantly enhances the transverse resonance frequency, their effect is negligible on the rotational resonance frequency.

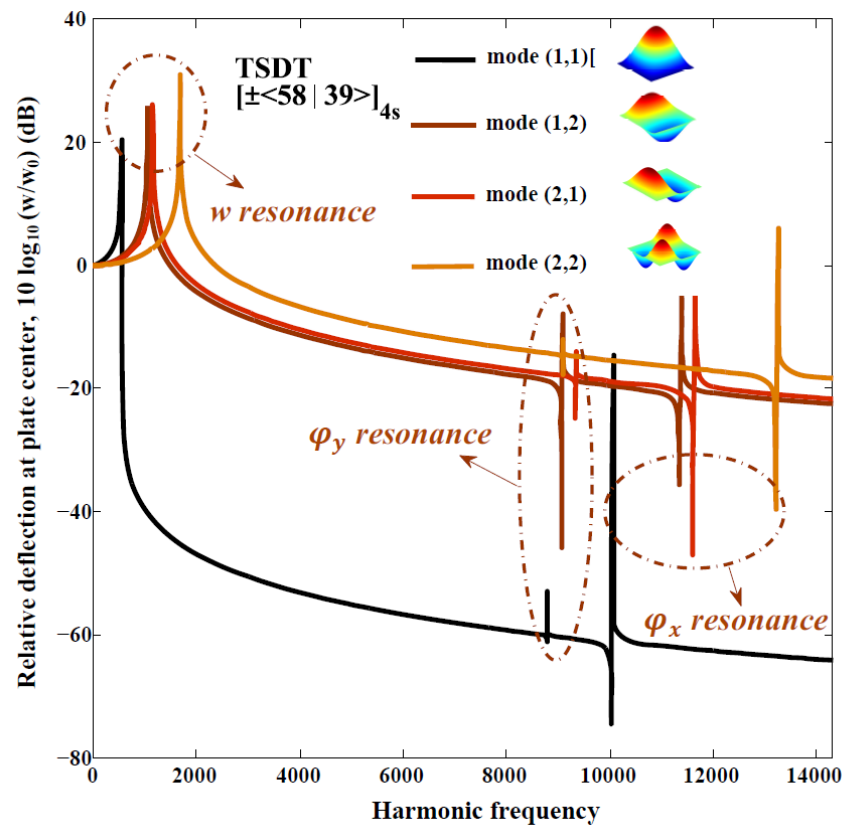

(a)

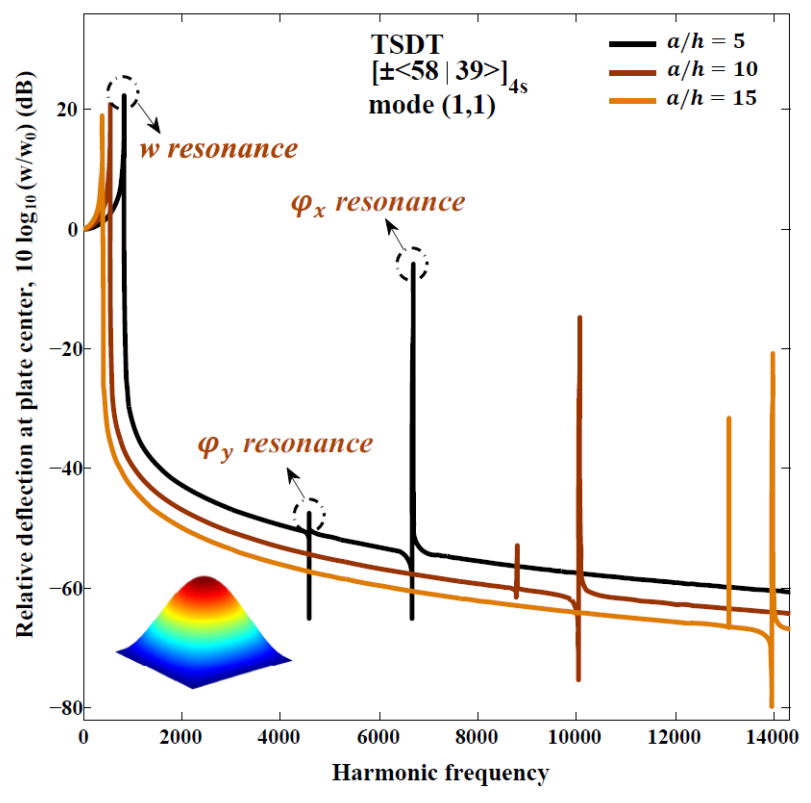

(b)

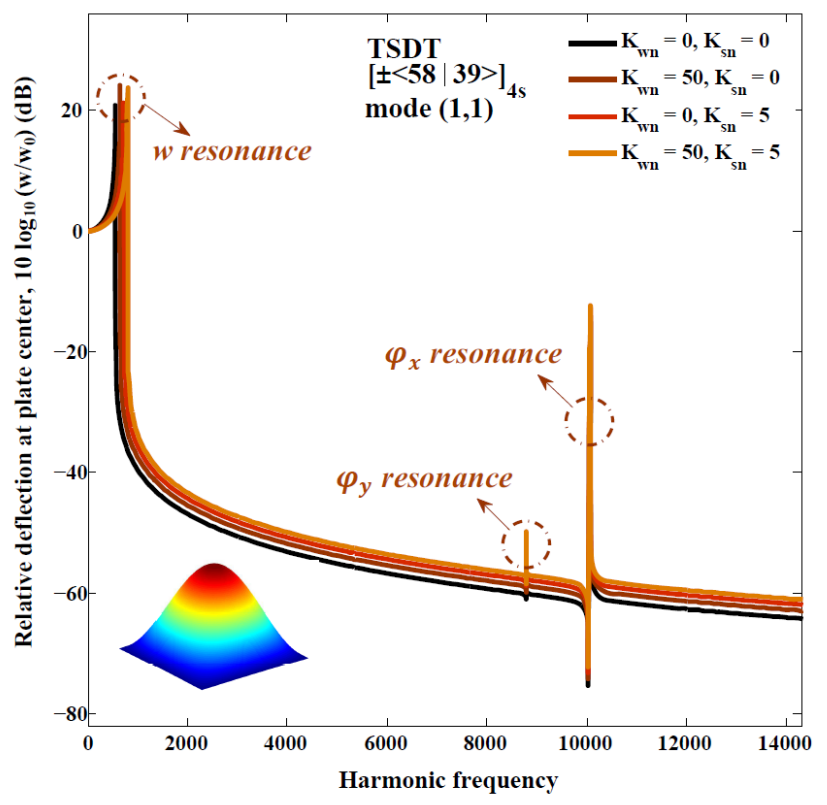

(c)

Fig. 6 Effect of (a) mode shape, (b) length-to-thickness ratio, and (c) elastic foundation on harmonic responses. 


\subsection{Optimum fiber path for vibratory variable stiffness composites}

Section 5.1 has shown the dynamic responses of fiber-steered composites with respect to three manufacturing scenarios. In this section, structural improvements are sought for a laminated plate resting on a Pasternak elastic foundation with $\left(K_{w n}, K_{s n}\right)=(50,5)$. The goal here is to find a trade-off among three antagonist objective functions that involve the minimization of the amplitude of the undamped out-of-plane dynamic deflection $(w)$, the maximization of the undamped frequency $(\omega)$, and the in-plane stiffness $\left(E_{e q}\right)$. We search for the fiber path parameters, $T_{0}$ and $T_{1}$ (design variables of the optimization scheme), that minimize $w$, and maximize $\omega$ and $E_{e q}$ through the following formulation:

$$
\begin{aligned}
& \min _{x}\left\{w(\mathbf{x}), \frac{1}{\omega(\mathbf{x})}, \frac{1}{E_{e q}(\mathbf{x})}\right\} ; \mathbf{x}=\left(T_{0}, T_{1}\right)^{T} \\
& \text { s.t. }\left\{T_{0}, T_{1} \in\left[0^{\circ}, 90^{\circ}\right] \& R \geq 25 \mathrm{in}\right\}
\end{aligned}
$$

where $\mathbf{x}$ represents the design variable vector, and $R$ is the minimum turning radius of AFP, which is a manufacturing constraint. The design variables should be integer to respect fiber orientation constraints imposed by a typical AFP machine. The non-dominated sorting genetic algorithm-II (NSGA-II) [70] has been chosen to solve the optimization problem above. Since NSGA-II is a population-based algorithm that requires a large number of function evaluations to reach the Pareto solutions, we resort here to a surrogate model, i.e. the Radial Basis Function. This strategy significantly reduces the computational cost of the optimization process. For a more detailed description of this surrogate-based algorithm, the reader is referred to [10, 12, 53].

Figure 7 illustrates the Pareto solutions that simultaneously minimize the out-of-plane deflection

$(\widetilde{w})$ and maximize the in-plane stiffness $\left(\widetilde{E}_{e q}\right)$ and the frequency $(\widetilde{\omega})$. The numerical results have been normalized by the value of their counterpart quasi-isotropic laminate with layup: $[45 / 0 /-45 / 90]_{2 s}$. As expected, Fig. 7 shows that gaps deteriorate the performance of defectfree fiber-steered laminates, as opposed to overlaps, which improve it. Overlaps have a more pronounced impact on the laminate performance than gaps. The reason is that the gaps in a laminate are filled with resin and result in a constant thickness, while overlaps are thickness build-up. On an equal-weight basis, the performance of the plate is divided by the plate weight, which scales linearly with the overlap area. However, the plate flexural rigidity is proportional to the cube of the plate thickness. As a result for vibration reduction, a complete overlap strategy is more beneficial than that with complete gap. 
With respect to the fiber orientation, the results in Fig. 7 obtained for a simply-supported square plate show the following: $\pm 45^{\circ}$ fiber angles are optimal for maximum out-of-plane stiffness [48], whereas straight fibers along the loading direction, i.e. $0^{\circ}$, are the best for the maximum in-plane stiffness [53]. A curvilinear fiber path, on the other hand, allows for a trade-off between the two properties. At the plate center, where the plate is unsupported and the maximum out-of-plane deflection occurs, the highest in-plane stiffness is achieved with fibers oriented along the loading direction. At the plate edges where the plate is simply supported, the fiber orientation close to $45^{\circ}$ leads to a high out-of-plane stiffness. Hence, as shown in Fig. 7, a large proportion of fibers with orientation at the plate center close to $0^{\circ}$ favours the in-plane stiffness, whereas a large proportion of fibers with angles close to $45^{\circ}$ improves the out-of-plane stiffness of the plate.

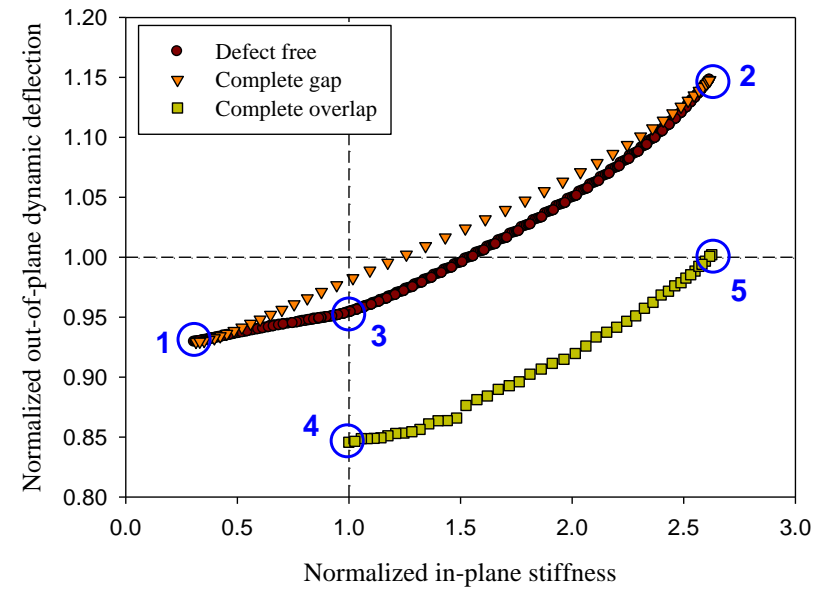

(a)

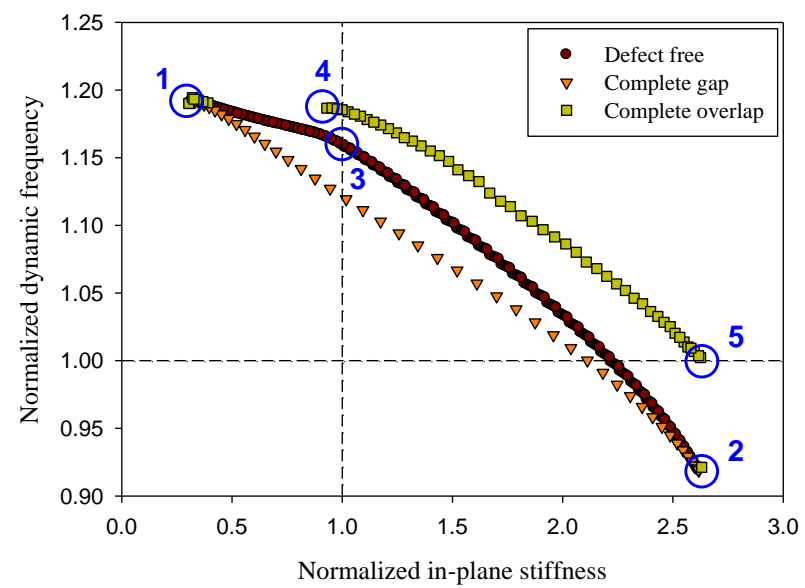

(b)

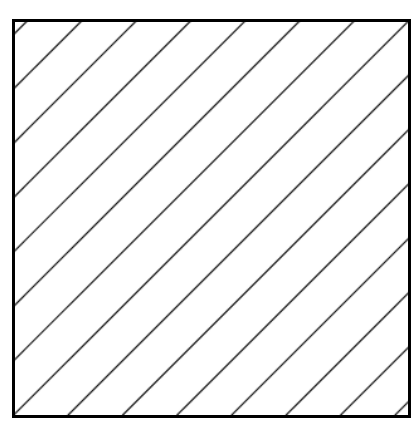

1) $[ \pm<45 \mid 45>]_{4 s}$

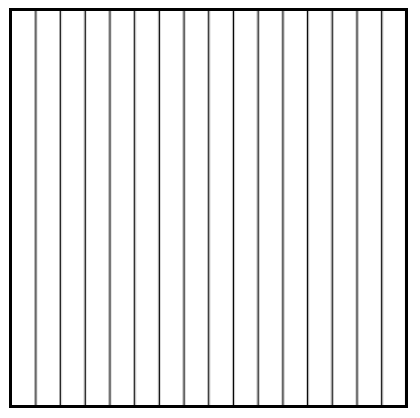

2) $[ \pm<0 \mid 0>]_{4 s}$

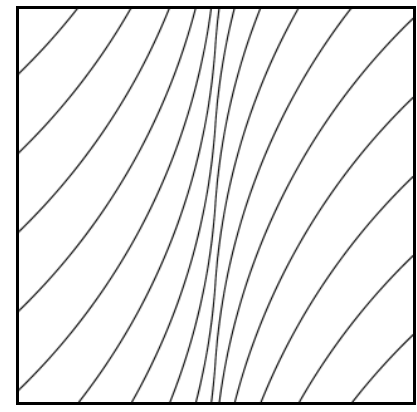

3) $[ \pm<4 \mid 46>]_{4 s}$ 


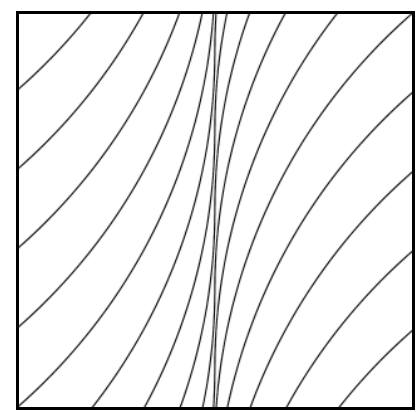

4) $[ \pm<0 \mid 49>]_{4 s}$

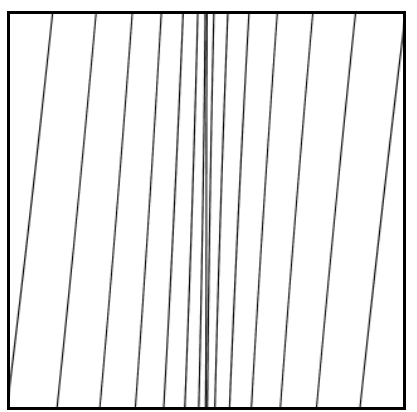

5) $[ \pm<0 \mid 7>]_{4 s}$

Fig. 7 Pareto fronts for (a) normalized out-of-plane dynamic deflection and (b) normalized frequency versus normalized in-plane stiffness.

\subsection{Vibration suppression via magnetostrictive layers}

In this section, we seek to actively reduce vibrations in the optimum plate $[ \pm<4 \mid 46>]_{4 s}$, i.e. point 3 in Fig.7, through the use of a pair of magnetostrictive layers. As shown in Fig. 2b, we consider a moderately-thick plate $\left(L /\left(h+2 h_{M}\right)=10\right)$ with active layers $\left(h_{M}=h / 20\right)$, one at the top and the other at the bottom of the sandwich panel. The material properties of the magnetostrictive layers are [1]:

$$
E_{m}=26.5 \mathrm{GPa}, v_{m}=0.0, \rho_{m}=9250 \mathrm{~kg} \cdot \mathrm{m}^{-3}, d_{m}=1.67 \times 10^{-8} \mathrm{~m} \cdot \mathrm{A}^{-1}
$$

We study the influence of the gain control parameter $\left(K_{c} C\right)$ and the elastic foundation constants on the damped out-of-plane deflection and velocity, damped angular frequency, and magnetostrictive damping coefficients. Figure 8a shows the damped out-of-plane midpoint deflection of a defect-free composite for several gain control parameters. In the absence of a gain control parameter $\left(K_{c} C=0\right)$, the transverse deflection shows undamped oscillatory responses around the static deflection. As soon as a gain control is applied, damped controlled responses appear. In particular, Fig. $8 \mathrm{~b}$ illustrates the amplitude versus velocity of the damped out-of-plane deflection for several gain control parameters, all starting from the zero initial condition. Increasing $K_{c} C$ enhances the attenuation rate of the transverse vibration amplitude and the velocity; these quantities finally approach the static transverse deflection and zero transverse deflection velocity for the damped dynamic responses. 


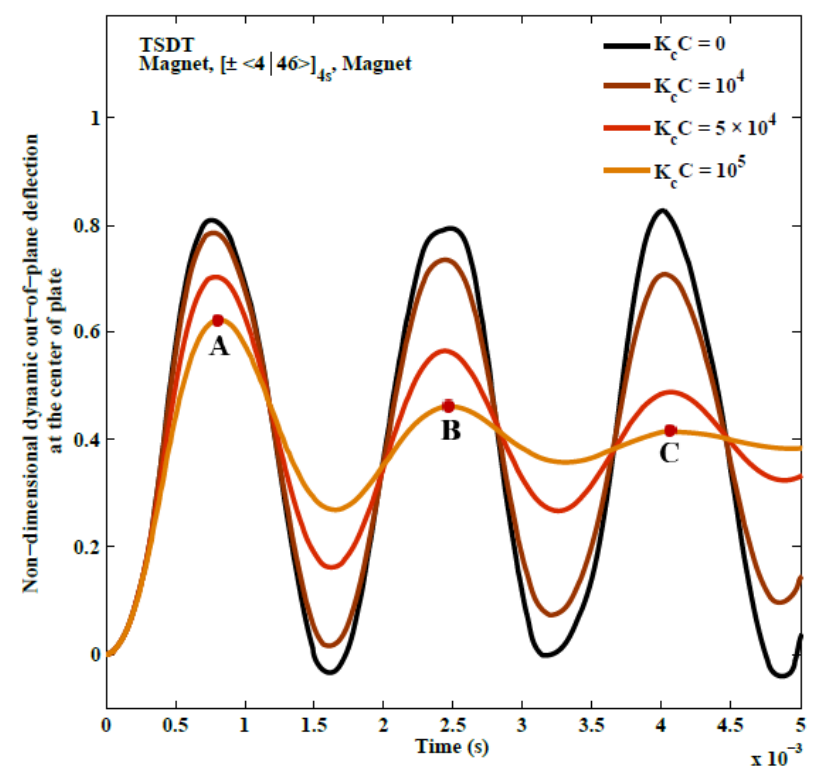

(a)

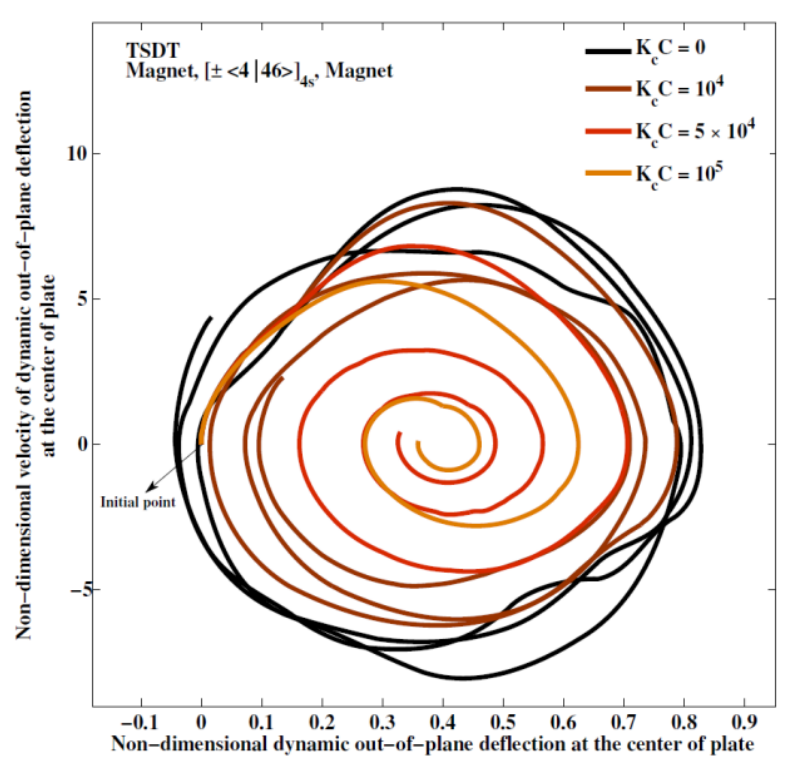

(b)

Fig. 8 (a) Time evolution of suppressed dynamic out-of-plane deflection $(\bar{w} \times 100)$ (Marked points are: A $\left(\bar{w}=0.62 \times 10^{-2}, t=8.15 \times 10^{-4}\right), \mathrm{B}\left(\bar{w}=0.42 \times 10^{-2}, t=2.45 \times 10^{-4}\right)$, and $\left.\mathrm{C}\left(\bar{w}=0.42 \times 10^{-2}, t=4.13 \times 10^{-4}\right)\right)$ and (b) velocity-deflection $(\bar{w} \times 100)$ at the center of an actively controlled, defect-free, fiber-steered composite for several control gain parameters.

The damping and frequency parameters $\left(-\alpha+i \omega_{d}\right)$ of the transverse dynamic deflection are presented in Table 1, for given manufacturing scenarios, gain controls, and elastic foundation constants. The numerical results for the variable stiffness composite $\left([ \pm<4 \mid 46>]_{4 s}\right)$, shown in Fig.7, are presented for the first mode of vibration only; similar results obtained for the other vibration modes are not included here. As illustrated in Table 1, the application of an elastic foundation increases the damped frequency $\left(\omega_{d}\right)$, but has no remarkable impact on the damping parameter $(\alpha)$. For instance, the damped vibration frequency of a defect-free variable stiffness plate, with an elastic foundation $\left(K_{w n}, K_{s n}\right)=(50,5)$ and a gain control of $K_{c} C=10^{4}$, is $51 \%$ higher than its counterpart which has no elastic foundation interaction $\left(K_{w n}, K_{s n}\right)=(0,0)$. The numerical results show that the gain control has a significant effect on the vibration attenuation while its effect on the damped vibration is negligible. Moreover, the vibration attenuation of a plate with gaps is more responsive to the gain control than that of a plate with overlaps.

Table 1 Eigenvalue parameters for vibration suppression control $\left(\lambda=-\alpha+i \omega_{d}\right)$ for prescribed manufacturing defects and elastic foundation parameters. 


\begin{tabular}{|c|c|c|c|c|c|c|c|c|}
\hline \multirow{2}{*}{$\begin{array}{l}\text { Foundation } \\
\text { constants }\end{array}$} & \multirow{2}{*}{$\begin{array}{c}\text { Manufacturing } \\
\text { defects }\end{array}$} & \multirow{2}{*}{$\begin{array}{l}K_{c} C=0 \\
\omega_{d} \times 10^{3}\end{array}$} & \multicolumn{2}{|c|}{$K_{c} C=10^{4}$} & \multicolumn{2}{|c|}{$K_{c} C=5 \times 10^{4}$} & \multicolumn{2}{|c|}{$K_{c} C=10^{5}$} \\
\hline & & & $\alpha \times 10^{2}$ & $\omega_{d} \times 10^{3}$ & $\alpha \times 10^{2}$ & $\omega_{d} \times 10^{3}$ & $\alpha \times 10^{2}$ & $\omega_{d} \times 10^{3}$ \\
\hline \multirow{3}{*}{$\begin{array}{l}K_{w n}=0 \\
K_{s n}=0\end{array}$} & Defect-free & 2.57 & 0.74 & 2.56 & 3.70 & 2.54 & 7.38 & 2.46 \\
\hline & Complete gap & 2.45 & 0.79 & 2.45 & 3.93 & 2.42 & 7.86 & 2.32 \\
\hline & $\begin{array}{c}\text { Complete } \\
\text { overlap }\end{array}$ & 2.94 & 0.61 & 2.94 & 3.04 & 2.92 & 6.08 & 2.87 \\
\hline \multirow{3}{*}{$\begin{array}{l}K_{w n}=50 \\
K_{s n}=0\end{array}$} & Defect-free & 3.07 & 0.74 & 3.07 & 3.70 & 3.05 & 7.39 & 2.98 \\
\hline & Complete gap & 2.99 & 0.79 & 2.99 & 3.94 & 2.97 & 7.87 & 2.89 \\
\hline & $\begin{array}{l}\text { Complete } \\
\text { overlap }\end{array}$ & 3.34 & 0.61 & 3.34 & 3.04 & 3.32 & 6.08 & 3.28 \\
\hline \multirow{3}{*}{$\begin{array}{l}K_{w n}=0 \\
K_{s n}=5\end{array}$} & Defect-free & 3.49 & 0.74 & 3.49 & 3.70 & 3.47 & 7.40 & 3.41 \\
\hline & Complete gap & 3.44 & 0.79 & 3.43 & 3.94 & 3.42 & 7.88 & 3.35 \\
\hline & $\begin{array}{l}\text { Complete } \\
\text { overlap }\end{array}$ & 3.69 & 0.61 & 3.69 & 3.04 & 3.67 & 6.09 & 3.63 \\
\hline \multirow{3}{*}{$\begin{array}{l}K_{w n}=50 \\
K_{s n}=5\end{array}$} & Defect-free & 3.88 & 0.74 & 3.88 & 3.71 & 3.86 & 7.40 & 3.81 \\
\hline & Complete gap & 3.84 & 0.79 & 3.84 & 3.95 & 3.82 & 7.88 & 3.76 \\
\hline & $\begin{array}{c}\text { Complete } \\
\text { overlap }\end{array}$ & 4.01 & 0.61 & 4.01 & 3.05 & 4.00 & 6.09 & 3.97 \\
\hline
\end{tabular}

\section{Concluding remarks}

This paper has examined the vibration response of sandwich plates with fiber-steered composite skins. The structural and vibration responses of the plates have been predicted with TSDT to account for the effect of transverse shear deformation in moderately-thick laminates. The results of forced and harmonic vibration analyses have shown a higher amplitude and a lower frequency for the dynamic out-of-plane deflection of a defect-free plate, as opposed to a plate with overlaps. We have modelled the interaction between the soft core and the fiber-steered laminated 
skins via a Pasternak elastic foundation. To concurrently improve the in-plane stiffness along with the vibration responses of sandwich panels, we have first obtained optimal fiber paths using a surrogate-based, multi-objective optimization algorithm. For simply-supported boundary conditions, we have found that a large proportion of fibers with orientation at the plate center close to $0^{\circ}$ favours the in-plane stiffness, whereas a large proportion of fibers with angles close to $45^{\circ}$ improves the out-of-plane stiffness and vibration responses. Second, we have introduced magnetostrictive layers on the top and bottom of the optimal laminates to actively improve the vibratory characteristics. It has been shown that vibration attenuation is more pronounced for a higher value of the gain control in the active layers. The methodology presented in this paper could be used in aerospace applications for health monitoring and vibration control of sandwich panels.

\section{Acknowledgments}

A.H. Akbarzadeh acknowledges the financial support from the Natural Sciences and Engineering Research Council of Canada (NSERC).

\section{References}

[1] Reddy JN. Mechanics of laminated composite plates and shells: theory and analysis: CRC press; 2004.

[2] Vel SS, Batra R. Analytical solution for rectangular thick laminated plates subjected to arbitrary boundary conditions. AIAA journal. 1999;37(11):1464-73.

[3] Bekas D, Tsirka K, Baltzis D, Paipetis A. Self-healing materials: A review of advances in materials, evaluation, characterization and monitoring techniques. Composites Part B: Engineering. 2016;87:92-119.

[4] Gloria A, Ronca D, Russo T, D'Amora U, Chierchia M, De Santis R, et al. Technical features and criteria in designing fiber-reinforced composite materials: From the aerospace and aeronautical field to biomedical applications. J Appl Biomater Biomech. 2011;9(2):151-63.

[5] de la Rosa García P, Escamilla AC, García MNG. Analysis of the flexural stiffness of timber beams reinforced with carbon and basalt composite materials. Composites Part B: Engineering. 2016;86:152-9.

[6] Arachchige B, Ghasemnejad H, Augousti A. Theoretical approach to predict transverse impact response of variable-stiffness curved composite plates. Composites Part B: Engineering. 2016;89:34-43.

[7] Reddy J, Miravete A. Practical analysis of composite laminatesCRC Press. Boca Raton. 1995.

[8] Gürdal Z, Tatting BF, Wu KC. Tow-placement technology and fabrication issues for laminated composite structures. 46th AIAA/ASME/ASCE/AHS/ASC Structures, Structural Dynamics \& Materials Conference. Austin, Texas, USA2005. p. 1-17. 
[9] Akhavan H, Ribeiro P, de Moura M. Large deflection and stresses in variable stiffness composite laminates with curvilinear fibres. International Journal of Mechanical Sciences. 2013;73:14-26.

[10] Arian Nik M, Fayazbakhsh K, Pasini D, Lessard L. Optimization of variable stiffness composites with embedded defects induced by Automated Fiber Placement. Composite Structures. 2014;107:160-6.

[11] Fayazbakhsh K, Arian Nik M, Pasini D, Lessard L. Defect layer method to capture effect of gaps and overlaps in variable stiffness laminates made by automated fiber placement. Composite Structures. 2013;97:245-51.

[12] Arian Nik M, Fayazbakhsh K, Pasini D, Lessard L. A comparative study of metamodeling methods for the design optimization of variable stiffness composites. Composite Structures. 2014; 107:494-501.

[13] Van Campen JM, Kassapoglou C, Gürdal Z. Generating realistic laminate fiber angle distributions for optimal variable stiffness laminates. Composites Part B: Engineering. 2012;43(2):354-60.

[14] Rouhi M, Ghayoor H, Hoa SV, Hojjati M. Multi-objective design optimization of variable stiffness composite cylinders. Composites Part B: Engineering. 2015;69:249-55.

[15] Akhavan H, Ribeiro P. Natural modes of vibration of variable stiffness composite laminates with curvilinear fibers. Composite Structures. 2011;93(11):3040-7.

[16] Khani A, IJsselmuiden S, Abdalla M, Gürdal Z. Design of variable stiffness panels for maximum strength using lamination parameters. Composites Part B: Engineering. 2011;42(3):546-52.

[17] Cairns D, Ilcewicz L, Walker T. Response of automated tow placed laminates to stress concentrations. NASA Langley Research Center, Third NASA Advanced Composites Technology Conference1993.

[18] Nik MA, Fayazbakhsh K, Pasini D, Lessard L. Optimization of variable stiffness composites with embedded defects induced by Automated Fiber Placement. Composite Structures. 2014;107:160-6.

[19] Nik Mahdi A, Lessard L, Pasini D. Size-dependent behavior of laminates with curvilinear fibers made by automated fiber placement. Science and Engineering of Composite Materials2015. p. 157.

[20] Falcó O, Mayugo J, Lopes C, Gascons N, Turon A, Costa J. Variable-stiffness composite panels: As-manufactured modeling and its influence on the failure behavior. Composites Part B: Engineering. 2014;56:660-9.

[21] George T, Deshpande VS, Wadley HN. Mechanical response of carbon fiber composite sandwich panels with pyramidal truss cores. Composites Part A: Applied Science and Manufacturing. 2013;47:31-40.

[22] Du Y, Yan N, Kortschot MT. Light-weight honeycomb core sandwich panels containing biofiber-reinforced thermoset polymer composite skins: fabrication and evaluation. Composites Part B: Engineering. 2012;43(7):2875-82.

[23] Airborne. Case study: Fiber steering, Study of a stowed solar array wing. http://airborneaerospace.com/544/2013.

[24] Frostig Y. Classical and high-order computational models in the analysis of modern sandwich panels. Composites part B: Engineering. 2003;34(1):83-100.

[25] Thomsen OT. Analysis of local bending effects in sandwich plates with orthotropic face layers subjected to localised loads. Composite Structures. 1993;25(1):511-20. 
[26] Kiani Y, Akbarzadeh A, Chen Z, Eslami M. Static and dynamic analysis of an FGM doubly curved panel resting on the Pasternak-type elastic foundation. Composite Structures. 2012;94(8):2474-84.

[27] Mansouri M, Shariyat M. Biaxial thermo-mechanical buckling of orthotropic auxetic FGM plates with temperature and moisture dependent material properties on elastic foundations. Composites Part B: Engineering. 2015;83:88-104.

[28] Straughan WT. Analysis of plates on elastic foundations. 1990.

[29] Naik N. Composite beams on elastic foundations. Journal of Thermoplastic Composite Materials. 2000;13(1):2-11.

[30] Agnes G, Mall S. Structural integrity issues during piezoelectric vibration suppression of composite structures. Composites Part B: Engineering. 1999;30(7):727-38.

[31] Kalamkarov A, Georgiades A. Modeling of smart composites on account of actuation, thermal conductivity and hygroscopic absorption. Composites Part B: Engineering. 2002;33(2):141-52.

[32] Li F-M, Lyu X-X. Active vibration control of lattice sandwich beams using the piezoelectric actuator/sensor pairs. Composites Part B: Engineering. 2014;67:571-8.

[33] Park J-M, Kim D-S, Han S-B. Properties of interfacial adhesion for vibration controllability of composite materials as smart structures. Composites science and technology. 2000;60(10):1953-63.

[34] Reddy J, Barbosa J. On vibration suppression of magnetostrictive beams. Smart materials and structures. 2000;9(1):49.

[35] Anjanappa M, Bi J. A theoretical and experimental study of magnetostrictive miniactuators. Smart Materials and Structures. 1994;3(2):83.

[36] Qian W, Liu G, Chun L, Lam K. Active vibration control of composite laminated cylindrical shells via surface-bonded magnetostrictive layers. Smart materials and structures. 2003;12(6):889.

[37] Pradhan S, Ng T, Lam K, Reddy J. Control of laminated composite plates using magnetostrictive layers. Smart materials and structures. 2001;10(4):657.

[38] Ghosh D, Gopalakrishnan S. Coupled analysis of composite laminate with embedded magnetostrictive patches. Smart materials and structures. 2005;14(6):1462.

[39] Cugnoni J, Gmür T, Schorderet A. Identification by modal analysis of composite structures modelled with FSDT and HSDT laminated shell finite elements. Composites Part A: Applied Science and Manufacturing. 2004;35(7):977-87.

[40] Ribeiro P. Forced periodic vibrations of laminated composite plates by a p-version, first order shear deformation, finite element. Composites science and technology. 2006;66(11):184456.

[41] Khdeir A, Reddy J. Exact solutions for the transient response of symmetric cross-ply laminates using a higher-order plate theory. Composites science and technology. 1989;34(3):205-24.

[42] Reddy J. Analysis of functionally graded plates. International Journal for Numerical Methods in Engineering. 2000;47(1-3):663-84.

[43] Akbarzadeh AH, Hosseini Zad SK, Eslami MR, Sadighi M. Mechanical behaviour of functionally graded plates under static and dynamic loading. Proceedings of the Institution of Mechanical Engineers, Part C: Journal of Mechanical Engineering Science. 2011;225(2):326-33. 
[44] Komeili A, Akbarzadeh AH, Doroushi A, Eslami MR. Static analysis of functionally graded piezoelectric beams under thermo-electro-mechanical loads. Advances in Mechanical Engineering. 2011;2011.

[45] Kulkarni SA, Bajoria KM. Large deformation analysis of piezolaminated smart structures using higher-order shear deformation theory. Smart Materials and Structures. 2007;16(5):1506.

[46] Asadi H, Akbarzadeh A, Wang Q. Nonlinear thermo-inertial instability of functionally graded shape memory alloy sandwich plates. Composite Structures. 2015;120:496-508.

[47] Asadi H, Akbarzadeh AH, Chen ZT, Aghdam MM. Enhanced thermal stability of functionally graded sandwich cylindrical shells by shape memory alloys. Smart Materials and Structures. 2015;24(4):045022.

[48] Akbarzadeh A, Nik MA, Pasini D. The role of shear deformation in laminated plates with curvilinear fiber paths and embedded defects. Composite Structures. 2014;118:217-27.

[49] Nikrad S, Asadi H, Akbarzadeh A, Chen Z. On thermal instability of delaminated composite plates. Composite Structures. 2015;132:1149-59.

[50] Kim BC, Potter K, Weaver PM. Continuous tow shearing for manufacturing variable angle tow composites. Composites Part A: Applied Science and Manufacturing. 2012;43(8):1347-56.

[51] Gürdal Z, Tatting BF, Wu CK. Variable stiffness composite panels: Effects of stiffness variation on the in-plane and buckling response. Composites Part A: Applied Science and Manufacturing. 2008;39(5):911-22.

[52] Blom AW, Lopes CS, Kromwijk PJ, Gürdal Z, PP C. A theoretical model to study the influence of tow-drop areas on the stiffness and strength of variable-stiffness laminates. Journal of Composite Materials. 2009;43(5):403-25.

[53] Arian Nik M, Fayazbakhsh K, Pasini D, Lessard L. Surrogate-based multi-objective optimization of a composite laminate with curvilinear fibers. Composite Structures. 2012;94(8):2306-13.

[54] Arian Nik M. Surrogate-based multi-objective optimization of variable stiffness composites with embedded defects induced by Automated Fiber Placement. Montreal, Canada: McGill University; 2014.

[55] Selvadurai AP. Elastic analysis of soil-foundation interaction: Elsevier; 1979.

[56] Akbarzadeh AH, Abedini A, Chen ZT. Effect of micromechanical models on structural responses of functionally graded plates. Composite Structures. 2015;119(0):598-609.

[57] Akbarzadeh A, Abbasi M, Eslami M. Coupled thermoelasticity of functionally graded plates based on the third-order shear deformation theory. Thin-Walled Structures. 2012;53:141-55.

[58] Kim J, Reddy J. Analytical solutions for bending, vibration, and buckling of FGM plates using a couple stress-based third-order theory. Composite Structures. 2013;103:86-98.

[59] Zhang B, He Y, Liu D, Lei J, Shen L, Wang L. A size-dependent third-order shear deformable plate model incorporating strain gradient effects for mechanical analysis of functionally graded circular/annular microplates. Composites Part B: Engineering. 2015.

[60] Akbarzadeh AH, Chen ZC. Thermo-magneto-electro-elastic responses of rotating hollow cylinders. Mechanics of Advanced Materials and Structures. 2014;21(1):67-80.

[61] Akbarzadeh A, Pasini D. Multiphysics of multilayered and functionally graded cylinders under prescribed hygrothermomagnetoelectromechanical loading. Journal of Applied Mechanics. 2014;81(4):041018.

[62] Pradhan S. Vibration suppression of FGM shells using embedded magnetostrictive layers. International journal of solids and structures. 2005;42(9):2465-88. 
[63] Zhou H-M, Zhou Y-H. Vibration suppression of laminated composite beams using actuators of giant magnetostrictive materials. Smart materials and structures. 2007;16(1):198.

[64] Akbarzadeh AH, Abbasi M, Hosseini Zad SK, Eslami MR. Dynamic analysis of functionally graded plates using the hybrid Fourier-Laplace transform under thermomechanical loading. Meccanica. 2011;46(6):1373-92.

[65] Murty K. Vibration suppression of laminated composite beams using embedded magnetostrictive layers. Institution of Engineers(India), Journal. 1998;78:38-44.

[66] Akbarzadeh A, Chen Z. On the harmonic magnetoelastic behavior of a composite cylinder with an embedded polynomial eigenstrain. Composite Structures. 2013;106:296-305.

[67] Jafari-Talookolaei R-A, Kargarnovin MH, Ahmadian MT. Dynamic response of a delaminated composite beam with general lay-ups based on the first-order shear deformation theory. Composites Part B: Engineering. 2013;55:65-78.

[68] Avramidis I, Morfidis K. Bending of beams on three-parameter elastic foundation. International Journal of Solids and Structures. 2006;43(2):357-75.

[69] Salas M, Focke O, Herrmann AS, Lang W. Wireless Power Transmission for Structural Health Monitoring of Fiber-Reinforced-Composite Materials. IEEE Sensors Journal. 2014;14(7):2171-6.

[70] Deb K, Agrawal S, Pratap A, Meyarivan T. A fast elitist non-dominated sorting genetic algorithm for multi-objective optimization: NSGA-II. Lecture notes in computer science. 2000;1917:849-58. 